\title{
MHD instability of pressure-driven flow of a non-Newtonian fluid
}

\author{
B. M. Shankar ${ }^{1}$. Jai Kumar ${ }^{2}$ •I. S. Shivakumara ${ }^{3}$ · S. B. Naveen Kumar ${ }^{1}$
}

Received: 13 May 2019 / Accepted: 22 October 2019 / Published online: 1 November 2019

(c) Springer Nature Switzerland AG 2019

\begin{abstract}
The effect of a uniform vertical magnetic field on the stability of pressure-driven flow of an electrically conducting nonNewtonian fluid in an isothermal channel is numerically investigated using the Chebyshev collocation method. The nonNewtonian fluid is modeled by the couple stress fluid theory, which permits for polar effects and encountered regularly in liquids with very large molecules. It is established that Squire's theorem is valid and the modified Orr-Sommerfeld equation is derived by considering the fact that the magnetic Prandtl number $P_{m}$, for recognized electrically conducting liquids is too small. The triplets $\left(R_{c}, a_{c}, c_{c}\right)$, where $R_{c}$ is the critical Reynolds number, $a_{c}$ is the critical wave number and $c_{c}$ is the critical wave speed, are obtained for different values of couple stress parameter $\Lambda$ and the Hartman number $M$. It is found that increasing $M$ has a stabilizing effect on the system while an increase in the couple stress parameter shows twofold deeds. Individual aspects of the kinetic energy spectrum are observed and presented for different parametric values to obtain comprehensive information at the critical state of fluid flow.
\end{abstract}

Keywords Stability · Pressure-driven flow · Magnetic field · Couple stress fluid

\section{List of symbols}

$B_{x^{\prime}} B_{y}, B_{z}$

$B_{b}=B_{b x} / R P_{m}$

$C=c_{r}+i c_{i}$

$c_{r}$

$c_{i}$

$E_{c}$

$E_{d}$

$E_{M}$

$E_{s}$

$h$

$M=B_{0} h \sqrt{\sigma / \mu_{d}}$

$P=p+B^{2} / 2 \mu$

$P_{m}=\sigma \mu \nu$

$R=u_{0} h / v$

$u, v, w$

$u_{0}$

$t$

$(x, y, z)$
Components of magnetic induction $\vec{B}$

Basic magnetic induction

Wave speed

Phase velocity

Growth rate

Kinetic energy due to couple stress viscosity

Kinetic energy due to surface drag

Kinetic energy due to magnetic field

Kinetic energy due to the shear term

Thickness of the porous channel

Hartmann number

Total pressure

Magnetic Prandtl number

Reynolds number

Components of velocity vector $\vec{q}$

Average velocity

Time

Cartesian co-ordinates

\section{Greek symbols}

$a$

$\beta$

$\eta$

$\Lambda=h \sqrt{\mu_{d} / \eta}$

$\mu_{d}$

$\mu$

$v$

$\sigma$

$\phi$

$\psi$

\section{Subscripts}

$b \quad$ Basic state

c Critical
Horizontal wave number

Vertical wave number

Couple stress viscosity

Couple stress parameter

Dynamic viscosity

Magnetic permeability

Kinematic viscosity

Electrical conductivity

Velocity stream function

Magnetic stream function

Basic state
Critical

B. M. Shankar, bmshankar@pes.edu| ${ }^{1}$ Department of Mathematics, PES University, Bangalore 560085 , India. ${ }^{2}$ ISRO Space Application Centre, Ahmadabad 380 015, India. ${ }^{3}$ Department of Mathematics, Bangalore University, Bangalore 560056 , India. 


\section{Introduction}

The pressure-driven flow in an isothermal channel has preoccupied fluid dynamicists for centuries. The ubiquitous nature of turbulence and its inability to give a universal theory have led many to try conceiving of the transition from laminar state to turbulent as a simple problem. It has many applications in engineering, geophysics, meteorology, oceanography and so on. The studies undertaken by many authors on this subject are very collectible in the book by Drazin and Reid [1].

The stability of hydromagnetic Poiseuille flow has additionally gotten equivalent significance in the literature. Lock [2] initiated this study by assuming $P_{m}$ is exceptionally small and showed that the magnetic field has a stabilizing effect on the fluid flow. Later, Potter and Kutchey [3] re-examined the problem without any simplification and observed that the fluid flow becomes more stable as $P_{m}$ increases. Takashima [4] re-considered the problem explored by Potter and Kutchey [3] under the appropriate boundary conditions on the magnetic field perturbations. The Lock's arguments were supported by Lingwood and Alboussiere [5], who solved the problem numerically including all magnetic contributions and their results differed only a few percent from Lock's results. Using the multi-deck asymptotic approach, the effect of transverse magnetic field on the stability of Poiseuille flow at high Reynolds number was investigated by Makinde [6]. Proskurin and Sagalakov [7] analyzed the effect of longitudinal magnetic field on the stability of Poiseuille flow. Later, Makinde and Mhone [8] extended the work of Takashima [4] to an isotropic porous domain case and subsequently to an anisotropic porous domain by Shankar and Shivakumara [9]. In the past, the stability of conducting fluid flows in a channel in the presence of a uniform magnetic field has attracted the attention of a host of researchers (Takashima [10], Kaddeche et al. [11], Balyaev and Smorodin [12], Adesanya et al. [13], Hayat et al. [14], Hudoba et al. [15], Hudoba and Molokov [16], Bhatti et al. [17], Shankar et al. [18], ljaz et al. [19], Hassan et al. [20], Shankar et al. [21] and references therein).

The stability of forced convection in a fluid layer has been studied extensively. However, its counterpart for non-Newtonian fluids is in the much-to-be preferred state as these fluids bound to occur in many engineering applications. There exist various types of non-Newtonian fluids and the couple stress fluid, formulated by Stokes [22] based on micro-continuum theories, is one such non-Newtonian fluid which takes into account the size of fluid particles. These fluids are found to be useful in scientific and engineering applications. The effect of couple stresses on the stability of fluid flow was initially studied by Jain and Stokes [23] and its counterpart in a porous medium was examined by Shankar et al. [24].
In a slit channel with metrically astringent walls, the hydromagnetic flow of a couple stress fluid was investigated by Mekheimer [25]. The stability of free (natural) convection in a vertical couple stress fluid layer was investigated by Shankar et al. [18] while later this study was extended by Shankar et al. [26] and Shankar et al. [27] to include a uniform AC electric field and magnetic field, respectively. In addition, many researchers investigated the effect of couple stresses on fluid flows in different contexts (Rudraiah et al. [28], Shivakumar et al. [29], Turkyilmazoglu [30], Shivakumara and Naveen Kumar [31], Srinivasacharya and Rao [32], Ramesh [33], Tripathi et al. [34], Mahabaleshwar et al. [35], Layek and Pati [36], Nandal and Mahajan [37], Reddy et al. [38], Naveen Kumar et al. [39]).

To the best of our knowledge, the simultaneous presence of couple stresses and magnetic field on the pressure-driven stability of fluid flow in a channel is missing in the literature despite the study finds importance in biological fluid flows. The novelty of this paper is to unveil the validity of Squire's theorem in analyzing the stability characteristics of the couple stress fluid in a channel in the presence of a uniform vertical magnetic field and undertaking energy budget analysis to understand the physical mechanisms involved in the flow transition.

\section{Mathematical formulation}

We consider fully developed pressure-driven steady flow of an electrically conducting incompressible couple stress fluid in an isothermal horizontal channel of thickness $2 h$ (Fig. 1). For modeling this flow, a Cartesian coordinate system $(x, y, z)$ is used where the axes $x, y$ and $z$ denote the streamwise, spanwise, and wall-normal coordinates, respectively. The fluid layer is permeated by a uniform vertical magnetic induction $\vec{B}_{0}=\left(0,0, B_{0}\right)$. Following Stokes [22], Takashima [4] and Shankar et al. [27], the governing linear stability equations in the dimensionless form are

$\frac{\partial u}{\partial x}+\frac{\partial v}{\partial y}+\frac{\partial w}{\partial z}=0$

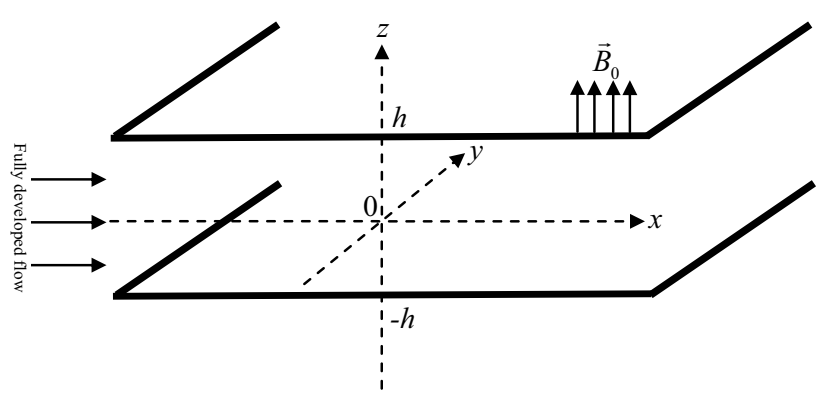

Fig. 1 Schematic of the problem considered 


$$
\begin{gathered}
\frac{\partial u}{\partial t}+u_{b} \frac{\partial u}{\partial x}+w \frac{\partial u_{b}}{\partial z}=-\frac{\partial P}{\partial x}+\frac{1}{R} \nabla^{2} u-\frac{1}{R \Lambda^{2}} \nabla^{4} u \\
+\frac{M^{2}}{R^{2} P_{m}}\left[\left(\frac{\partial}{\partial z}+R P_{m} B_{b} \frac{\partial}{\partial x}\right) B_{x}+R P_{m} B_{z} \frac{\partial B_{b}}{\partial z}\right]
\end{gathered}
$$

Equations (10) and (11) are solved using the boundary conditions

$u_{b}=u_{b}^{\prime \prime}=0, B_{x b}=0$ at $z= \pm 1$.

The solution is found to be

$$
\begin{aligned}
& u_{b}=\frac{-b^{2} \cosh \left(\frac{a z}{\sqrt{2}}\right) \cosh \left(\frac{b}{\sqrt{2}}\right)+\cosh \left(\frac{a}{\sqrt{2}}\right)\left[2 \sqrt{\Lambda^{4}-4 M^{2} \Lambda^{2}} \cosh \left(\frac{b}{\sqrt{2}}\right)+a^{2} \cosh \left(\frac{b z}{\sqrt{2}}\right)\right]}{-b^{2} \cosh \left(\frac{b}{\sqrt{2}}\right)+\cosh \left(\frac{a}{\sqrt{2}}\right)\left[a^{2}+2 \sqrt{\Lambda^{4}-4 M^{2} \Lambda^{2}} \cosh \left(\frac{b}{\sqrt{2}}\right)\right]} \\
& B_{x b}=\frac{\sqrt{2}\left[b^{3} \cosh \left(\frac{b}{\sqrt{2}}\right)\left(-z \sinh \left(\frac{a}{\sqrt{2}}\right)+\sinh \left(\frac{a z}{\sqrt{2}}\right)\right)+a^{3} \cosh \left(\frac{a}{\sqrt{2}}\right)\left(z \sinh \left(\frac{b}{\sqrt{2}}\right)-\sinh \left(\frac{b z}{\sqrt{2}}\right)\right)\right]}{a b\left[-b^{2} \cosh \left(\frac{b}{\sqrt{2}}\right)+\cosh \left(\frac{a}{\sqrt{2}}\right)\left(a^{2}+2 \sqrt{\Lambda^{4}-4 M^{2} \Lambda^{2}} \cosh \left(\frac{b}{\sqrt{2}}\right)\right)\right]}
\end{aligned}
$$

$\frac{\partial v}{\partial t}+u_{b} \frac{\partial v}{\partial x}=-\frac{\partial P}{\partial y}+\frac{1}{R} \nabla^{2} v-\frac{1}{R \Lambda^{2}} \nabla^{4} v+\frac{M^{2}}{R^{2} P_{m}}\left(\frac{\partial}{\partial z}+R P_{m} B_{b} \frac{\partial}{\partial x}\right) B_{y}$

$$
\begin{gathered}
\frac{\partial w}{\partial t}+u_{b} \frac{\partial w}{\partial x}=-\frac{\partial P}{\partial z}+\frac{1}{R} \nabla^{2} w-\frac{1}{R \Lambda^{2}} \nabla^{4} w \\
+\frac{M^{2}}{R^{2} P_{m}}\left(\frac{\partial}{\partial z}+R P_{m} B_{b} \frac{\partial}{\partial x}\right) B_{z}
\end{gathered}
$$

$\frac{\partial B_{x}}{\partial x}+\frac{\partial B_{y}}{\partial y}+\frac{\partial B_{z}}{\partial z}=0$

$$
\begin{aligned}
& \frac{\partial B_{x}}{\partial t}+u_{b} \frac{\partial B_{x}}{\partial x}+R P_{m} w \frac{\partial B_{b}}{\partial z}=\left(\frac{\partial}{\partial z}+R P_{m} B_{b} \frac{\partial}{\partial x}\right) u \\
& +B_{z} \frac{\partial u_{b}}{\partial z}+\frac{1}{R P_{m}} \nabla^{2} B_{x}
\end{aligned}
$$$$
\frac{\partial B_{y}}{\partial t}+u_{b} \frac{\partial B_{y}}{\partial x}=\left(\frac{\partial}{\partial z}+R P_{m} B_{b} \frac{\partial}{\partial x}\right) v+\frac{1}{R P_{m}} \nabla^{2} B_{y}
$$$$
\frac{\partial B_{z}}{\partial t}+u_{b} \frac{\partial B_{z}}{\partial x}=\left(\frac{\partial}{\partial z}+R P_{m} B_{b} \frac{\partial}{\partial x}\right) w+\frac{1}{R P_{m}} \nabla^{2} B_{z}
$$

The relevant boundary conditions are

$u=u^{\prime \prime}=v=v^{\prime \prime}=w=w^{\prime \prime}=0, B_{x}=B_{y}=\frac{\partial B_{z}}{\partial z}=0$ at $z= \pm 1$.

The basic flow is fully developed, unidirectional, steady and laminar. Under these circumstances, the governing equations reduce to

$\frac{d^{2} u_{b}}{d z^{2}}-\frac{1}{\Lambda^{2}} \frac{d^{4} u_{b}}{d z^{4}}+\frac{M^{2}}{R P_{m}} \frac{d B_{x b}}{d z}=R \frac{d P_{b}}{d x}$ where $a=\sqrt{\Lambda^{2}-\sqrt{\Lambda^{4}-4 M^{2} \Lambda^{2}}}, b=\sqrt{\Lambda^{2}+\sqrt{\Lambda^{4}-4 M^{2} \Lambda^{2}}}$. We note that $u_{b}=\frac{\cosh M-\cosh M z}{\cosh M-1}$ and $B_{x b}=\frac{\sinh M z-z \sinh M}{M(\cosh M-1)}$ as $\Lambda \rightarrow \infty$ which coincide with the expression obtained by Takashima [4]. Since $u_{b}$ and $B_{x b}$ are real, from Eqs. (13) and (14) it follows that $\Lambda>2 M$. We look for normal mode solution of Eqs. (1)-(8) in the form

$$
\begin{aligned}
(u, v, w, P) & =(\hat{u}, \hat{v}, \hat{w}, \hat{P})(z) e^{i(\alpha(x-c t)+\beta y)},\left(B_{x}, B_{y}, B_{z}\right) \\
& =R\left(\hat{B}_{x}, \hat{B}_{y}, \hat{B}_{z}\right)(z) e^{i(\alpha(x-c t)+\beta y)} .
\end{aligned}
$$

Substituting Eq. (15) into Eqs. (1)-(8), we obtain Using Eq. (15), Eqs. (1)-(8) become,

$$
\begin{aligned}
& D \hat{w}+i(\alpha \hat{u}+\beta \hat{v})=0 \\
& i \alpha\left(u_{b}-c\right) \hat{u}+\hat{w} D u_{b}=-i \alpha \hat{P}+\frac{1}{R}\left(D^{2}-\alpha^{2}-\beta^{2}\right) \hat{u} \\
& \quad-\frac{1}{R \Lambda^{2}}\left(D^{4}+\alpha^{4}+\beta^{4}+2 \alpha^{2} \beta^{2}-2 \alpha^{2} D^{2}-2 \beta^{2} D^{2}\right) \hat{u} \\
& \quad+M^{2}\left(\frac{D \hat{B}_{x}}{R P_{m}}+i \alpha B_{b} \hat{B}_{x}+\hat{B}_{z} D B_{b}\right)
\end{aligned}
$$

$$
\begin{aligned}
& i \alpha\left(u_{b}-c\right) \hat{v}=-i \beta \hat{P}+\frac{1}{R}\left(D^{2}-\alpha^{2}-\beta^{2}\right) \hat{v} \\
& -\frac{1}{R \Lambda^{2}}\left(D^{4}+\alpha^{4}+\beta^{4}+2 \alpha^{2} \beta^{2}-2 \alpha^{2} D^{2}-2 \beta^{2} D^{2}\right) \hat{v} \\
& \quad+M^{2}\left(\frac{D \hat{B}_{y}}{R P_{m}}+i \alpha B_{b} \hat{B}_{y}\right)
\end{aligned}
$$




$$
\begin{aligned}
& i \alpha\left(u_{b}-c\right) \hat{w}=-D \hat{P}+\frac{1}{R}\left(D^{2}-\alpha^{2}-\beta^{2}\right) \hat{w} \\
& \quad-\frac{1}{R \Lambda^{2}}\left(D^{4}+\alpha^{4}+\beta^{4}+2 \alpha^{2} \beta^{2}-2 \alpha^{2} D^{2}-2 \beta^{2} D^{2}\right) \hat{w} \\
& \quad+M^{2}\left(\frac{D \hat{B}_{z}}{R P_{m}}+i \alpha B_{b} \hat{B}_{z}\right) \\
& D \hat{B}_{z}+i\left(\alpha \hat{B}_{x}+\beta \hat{B}_{y}\right)=0 \\
& i \alpha R\left(u_{b}-c\right) \hat{B}_{x}+R P_{m} \hat{W} D B_{b}=\left(D \hat{u}+i \alpha R P_{m} B_{b} \hat{u}+R \hat{B}_{z} D u_{b}\right) \\
& \quad+\frac{1}{P_{m}}\left(D^{2}-\alpha^{2}-\beta^{2}\right) \hat{B}_{x}
\end{aligned}
$$

$i \alpha R\left(u_{b}-c\right) \hat{B}_{y}=\left(D \hat{v}+i \alpha R P_{m} B_{b} \hat{v}\right)+\frac{1}{P_{m}}\left(D^{2}-\alpha^{2}-\beta^{2}\right) \hat{B}_{y}$

$i \alpha R\left(u_{b}-c\right) \hat{B}_{z}=\left(D \hat{w}+i \alpha R P_{m} B_{b} \hat{w}\right)+\frac{1}{P_{m}}\left(D^{2}-\alpha^{2}-\beta^{2}\right) \hat{B}_{z}$

Let us introduce the following modified Squire's transformation
The relevant boundary conditions are

$\tilde{u}=D \tilde{u}=D^{3} \tilde{u}=\tilde{w}=0, D \tilde{B}_{z}=\tilde{B}_{x}=0$ at $z= \pm 1$.

It is viewed that Eqs. (25)-(30) and Eqs. (16)-(23) display the same mathematical structure if $\beta=v=B_{y}=0$. The transformation involving the Reynolds number suggests that $2 \mathrm{D}$ disturbances are more unstable than $3 \mathrm{D}$ ones. The velocity stream function $\phi(x, z, t)$ and the magnetic stream function $\psi(x, z, t)$ are introduced through (neglect the tilde in the subsequent analysis)

$u=\frac{\partial \phi}{\partial z}, w=-\frac{\partial \phi}{\partial x}, B_{x}=\frac{\partial \psi}{\partial z}, B_{z}=-\frac{\partial \psi}{\partial x}$

and eliminate $P$ from the Eqs. (26) and (27) to obtain

$$
\begin{aligned}
& \left(u_{b}-c\right)\left(D^{2}-\alpha^{2}\right) \phi-D^{2} u_{b} \phi \\
& \quad=\frac{1}{i \alpha R}\left(D^{2}-\alpha^{2}\right)^{2} \phi-\frac{1}{i \alpha R \Lambda^{2}}\left(D^{2}-\alpha^{2}\right)^{3} \phi \\
& +M^{2}\left[\frac{1}{i \alpha R P_{m}} D\left(D^{2}-\alpha^{2}\right) \psi+B_{b}\left(D^{2}-\alpha^{2}\right) \psi-D^{2} B_{b} \psi\right] \\
& \left(u_{b}-c\right) \psi=\left(\frac{1}{i \alpha R} D \phi+P_{m} B_{b} \phi\right)+\frac{1}{i \alpha R P_{m}}\left(D^{2}-\alpha^{2}\right) \psi .
\end{aligned}
$$

$\tilde{\alpha}=\sqrt{\alpha^{2}+\beta^{2}}, \quad \tilde{u}=\frac{\alpha \hat{u}+\beta \hat{V}}{\tilde{\alpha}}, \quad \tilde{w}=\hat{w}, \tilde{P}=\frac{\tilde{\alpha} \hat{P}}{\alpha}, \quad \tilde{B}_{x}=\frac{\alpha \hat{B}_{x}+\beta \hat{B}_{y}}{\tilde{\alpha}}, \tilde{B}_{z}=\hat{B}_{z}, \quad \tilde{R}=\frac{\alpha R}{\tilde{\alpha}}, \tilde{\Lambda}=\Lambda$,

$\tilde{M}=M, \tilde{P}_{m}=P_{m}$

so that the exceeding $3 \mathrm{D}$ equations can be reduced to an equivalent $2 \mathrm{D}$ ones.

Equations (16)-(20), using Eq. (24), become

$$
\begin{aligned}
& D \tilde{w}+i \tilde{\alpha} \tilde{u}=0 \\
& i \tilde{\alpha}\left(u_{b}-c\right) \tilde{u}+\tilde{w} D w_{b}=-i \tilde{\alpha} \tilde{P}+\frac{1}{\tilde{R}}\left(D^{2}-\tilde{\alpha}^{2}\right) \tilde{u} \\
& -\frac{1}{\tilde{R} \tilde{\Lambda}^{2}}\left(D^{2}-\tilde{\alpha}^{2}\right)^{2} \tilde{u}+\tilde{M}^{2}\left(\frac{D \tilde{B}_{x}}{\tilde{R} \tilde{P}_{m}}+i \tilde{\alpha} B_{b} \tilde{B}_{x}+\tilde{B}_{z} D B_{b}\right) \\
& i \tilde{\alpha}\left(u_{b}-c\right) \tilde{w}=-D \tilde{P}+\frac{1}{\tilde{R}}\left(D^{2}-\tilde{\alpha}^{2}\right) \tilde{w}-\frac{1}{\tilde{R} \tilde{\Lambda}^{2}}\left(D^{2}-\tilde{\alpha}^{2}\right)^{2} \tilde{w} \\
& +\tilde{M}^{2}\left(\frac{D \tilde{B}_{z}}{\tilde{R} \tilde{P}_{m}}+i \tilde{\alpha} B_{b} \tilde{B}_{z}\right) \\
& D \tilde{B}_{z}+i \tilde{\alpha} \tilde{B}_{x}=0 \\
& i \tilde{\alpha} \tilde{R}\left(u_{b}-c\right) \tilde{B}_{x}+\tilde{R} \tilde{P}_{m} \tilde{W} D B_{b}=\left(D \tilde{u}+i \tilde{\alpha} \tilde{R} \tilde{P}_{m} B_{b} \tilde{u}+\tilde{R} \tilde{B}_{z} D u_{b}\right) \\
& +\frac{1}{\tilde{P}_{m}}\left(D^{2}-\tilde{\alpha}^{2}\right) \tilde{B}_{x}
\end{aligned}
$$

$i \tilde{\alpha} \tilde{R}\left(u_{b}-c\right) \tilde{B}_{z}=\left(D \tilde{W}+i \tilde{\alpha} \tilde{R} \tilde{P}_{m} B_{b} \tilde{w}\right)+\frac{1}{\tilde{P}_{m}}\left(D^{2}-\tilde{\alpha}^{2}\right) \tilde{B}_{z}$.
It is well known that $P_{m}$ for the liquid sodium is of the order $10^{-5}$ and for mercury it is of the order $10^{-7}$ and hence terms in Eqs. (33) and (34) concerning $B_{b}$, and $\left(u_{b}-c\right) \psi$ in Eq. (34) can be neglected, provided that $R$ is not too large. As a result, the above equations lead to a single stability equation

$$
\begin{aligned}
& \left(u_{b}-c\right)\left(D^{2}-\alpha^{2}\right) \phi-\left(D^{2} u_{b}\right) \phi \\
& \quad=\frac{1}{i \alpha R}\left[\left(D^{2}-\alpha^{2}\right)^{2} \phi-\frac{1}{\Lambda^{2}}\left(D^{2}-\alpha^{2}\right)^{3} \phi-M^{2} D^{2} \phi\right] .
\end{aligned}
$$

Equation (35) agrees with Takashima [4] for the Newtonian case $(\Lambda \rightarrow \infty)$.

The boundary conditions on the magnetic field are no longer required now and the suitable boundary conditions are

$\phi=D \phi=D^{3} \phi=0 \quad$ at $\quad z= \pm 1$.

\section{Numerical solution}

The resulting stability equation is solved using the Chebyshev collocation method, which is an efficient and fast numerical solver for the investigation of the stability of fluid flow problems. We follow the procedure given in 
Shankar et al. [27] to discretize the stability equation, which yields
The exceeding equations form the following system of linear algebraic equation

$$
\begin{aligned}
& i \alpha R\left(\left(u_{b}-c\right)\left(\left(1-z_{j}^{2}\right)^{2} \sum_{m=0}^{N} B_{j m} \phi_{m}-8 z_{j}\left(1-z_{j}^{2}\right) \sum_{m=0}^{N} A_{j m} \phi_{m}+\left(8 z_{j}^{2}-4\left(1-z_{j}^{2}\right)\right) \phi_{j}\right)-\alpha^{2}\left(u_{b}-c\right) \phi_{j}-\frac{d^{2} u_{b}}{d x^{2}} \phi_{j}\right) \\
& =\left(\left(z_{j}^{2}-1\right)^{2} \sum_{m=0}^{N} E_{j m} \phi_{m}+16 z_{j}\left(z_{j}^{2}-1\right) \sum_{m=0}^{N} C_{j m} \phi_{m}+24\left(3 z_{j}^{2}-1\right) \sum_{m=0}^{N} B_{j m} \phi_{m}+96 z_{j} \sum_{m=0}^{N} A_{j m} \phi_{m}+24 \phi_{j}\right) \\
& +\alpha^{4} \phi_{j}-2 \alpha^{2}\left(\left(1-z_{j}^{2}\right)^{2} \sum_{m=0}^{N} B_{j m} \phi_{m}-8 z_{j}\left(1-z_{j}^{2}\right) \sum_{k=0}^{N} A_{j m} \phi_{m}+\left(8 z_{j}^{2}-4\left(1-z_{j}^{2}\right)\right) \phi_{j}\right) \\
& -\frac{1}{\Lambda^{2}}\left\{\begin{array}{c}
\left(z_{j}^{2}-1\right)^{2} \sum_{m=0}^{N} G_{j m} \phi_{m}+24 z_{j}\left(z_{j}^{2}-1\right) \sum_{m=0}^{N} F_{j m} \phi_{m} \\
+60\left(3 z_{j}^{2}-1\right) \sum_{m=0}^{N} E_{j m} \phi_{m}+480 z_{j} \sum_{m=0}^{N} C_{j m} \phi_{m}+360 \sum_{k=0}^{N} B_{j m} \phi_{m} \\
-3 \alpha^{2}\left(\begin{array}{c}
\left(z_{j}^{2}-1\right)^{2} \sum_{m=0}^{N} E_{j m} \phi_{m}+16 z_{j}\left(z_{j}^{2}-1\right) \sum_{m=0}^{N} C_{j m} \phi_{m}+24\left(3 z_{j}^{2}-1\right) \sum_{m=0}^{N} B_{j m} \phi_{m} \\
+96 z_{j} \sum_{m=0}^{N} A_{j m} \phi_{m}+24 \phi_{j}
\end{array}\right) \\
+3 \alpha^{4}\left(\left(1-z_{j}^{2}\right)^{2} \sum_{m=0}^{N} B_{j m} \phi_{m}-8 z_{j}\left(1-z_{j}^{2}\right) \sum_{m=0}^{N} A_{j m} \phi_{m}+\left(8 z_{j}^{2}-4\left(1-z_{j}^{2}\right)\right) \phi_{j}\right)-\alpha^{6} \phi_{j}
\end{array}\right\} \\
& \left(\begin{array}{c}
\left.\left.1-z_{j}^{2}\right)^{2} \sum_{m=0}^{N} B_{j m} \phi_{m}-8 z_{j}\left(1-z_{j}^{2}\right) \sum_{m=0}^{N} A_{j m} \phi_{m}+\left(8 z_{j}^{2}-4\left(1-z_{j}^{2}\right)\right) \phi_{j}\right), j=1(1) N-1
\end{array}\right\}
\end{aligned}
$$

with the corresponding boundary conditions

$$
\underline{\Delta_{1}} X=c \Delta_{2} X
$$

$\left(1-z_{j}^{2}\right)^{2} \sum_{m=0}^{N} C_{j m} \phi_{m}-12 z_{j}\left(1-z_{j}^{2}\right) \sum_{m=0}^{N} B_{j m} \phi_{m}-12\left(1-3 z_{j}^{2}\right) \sum_{m=0}^{N} A_{j m} \phi_{m}+24 z_{j} \phi_{j}=0, j=0 \& N$

where (Canuto et al. [40])

$A_{j k}= \begin{cases}\frac{c_{j}(-1)^{m+j}}{c_{m}\left(z_{j}-z_{m}\right)} & j \neq m \\ \frac{z_{j}}{2\left(1-z_{j}^{2}\right)} & 1 \leq j=m \leq N-1 \\ \frac{2 N^{2}+1}{6} & j=m=0 \\ -\frac{2 N^{2}+1}{6} & j=m=N\end{cases}$ where $c$ is the eigenvalue and $X$ is the discrete representation of eigenfunctions; $\Delta_{1}$ and $\Delta_{2}$ are matrices of order $(N+1)$. For fastened values of all parameters concerned within, the eigenvalue $c$ which compose a definite nontrivial solution of Eq. (41) is reached. Once $c$ is established, then the method and algorithm explained in Shankar et al. [41] are followed to find the critical parameters.

$B_{j m}=A_{j k} \cdot A_{k m}, \quad C_{j m}=B_{j k} \cdot A_{k m}, \quad E_{j m}=C_{j k} \cdot A_{k m}, \quad F_{j m}=E_{j k} \cdot A_{k m} \quad G_{j m}=F_{j k} \cdot A_{k m}$

with

$c_{j}=\left\{\begin{array}{ll}2 & j=0, N \\ 1 & 1 \leq j \leq N-1\end{array}\right.$. 


\section{Energy analysis}

To understand the physical mechanisms that contribute to instability, the two-dimensional kinetic energy balances are studied. The rate of change of kinetic energy in dimensionless form is given by strongly modify the stability of the system. Figures $2 a, b$ and $3 a, b$ display the impact of Hartman number and couple stress parameter on $u_{b}$ and $B_{b}$, respectively. Figure $2 a$, $b$, respectively exhibit that raise in the values of $M$ and $A$ is to boost the fluid flow. Figure $3 \mathrm{a}, \mathrm{b}$ show that $B_{b}$ is concealed with rising $M$ (Fig. 3a) and $\Lambda$ (Fig. 3b).

$$
\begin{aligned}
& \frac{1}{2} \frac{\partial}{\partial t}\left\langle u^{2}+v^{2}+w^{2}\right\rangle=-\left\langle u w \frac{d u_{b}}{d z}\right\rangle-\frac{1}{R}\left\langle\left(\left(\frac{\partial u}{\partial x}\right)^{2}+\left(\frac{\partial u}{\partial y}\right)^{2}+\left(\frac{\partial u}{\partial z}\right)^{2}\right)+\left(\left(\frac{\partial w}{\partial x}\right)^{2}+\left(\frac{\partial w}{\partial y}\right)^{2}+\left(\frac{\partial w}{\partial z}\right)^{2}\right)\right\rangle \\
& -\frac{1}{R \Lambda^{2}}\left\langle\left(\begin{array}{c}
u\left(\frac{\partial^{4}}{\partial x^{4}}+\frac{\partial^{4}}{\partial y^{4}}+\frac{\partial^{4}}{\partial z^{4}}+2 \frac{\partial^{4}}{\partial x^{2} \partial y^{2}}+2 \frac{\partial^{4}}{\partial x^{2} \partial z^{2}}+2 \frac{\partial^{4}}{\partial y^{2} \partial z^{2}}\right) u+ \\
v\left(\frac{\partial^{4}}{\partial x^{4}}+\frac{\partial^{4}}{\partial y^{4}}+\frac{\partial^{4}}{\partial z^{4}}+2 \frac{\partial^{4}}{\partial x^{2} \partial y^{2}}+2 \frac{\partial^{4}}{\partial x^{2} \partial z^{2}}+2 \frac{\partial^{4}}{\partial y^{2} \partial z^{2}}\right) v+ \\
w\left(\frac{\partial^{4}}{\partial x^{4}}+\frac{\partial^{4}}{\partial y^{4}}+\frac{\partial^{4}}{\partial z^{4}}+2 \frac{\partial^{4}}{\partial x^{2} \partial y^{2}}+2 \frac{\partial^{4}}{\partial x^{2} \partial z^{2}}+2 \frac{\partial^{4}}{\partial y^{2} \partial z^{2}}\right) w
\end{array}\right)\right\rangle-\frac{M^{2}}{R}\left\langle u^{2}\right\rangle \\
& =E_{s}+E_{d}+E_{c}+E_{M} \text {. }
\end{aligned}
$$

Here, the brackets \langle\rangle represent the volumetric average over the volume of disturbance waves.

\section{Results and discussion}

The stability against small disturbances of the pressuredriven plane laminar motion of an electrically conducting couple stress fluid under a transverse magnetic field is investigated numerically using the Chebyshev collocation method. A necessary condition for the amplification of a disturbance in the basic flow field is found to be $\Lambda>2 M$. We begin the presentation by demonstrating the basic velocity $u_{b}$ and the basic magnetic induction $B_{b}$, which

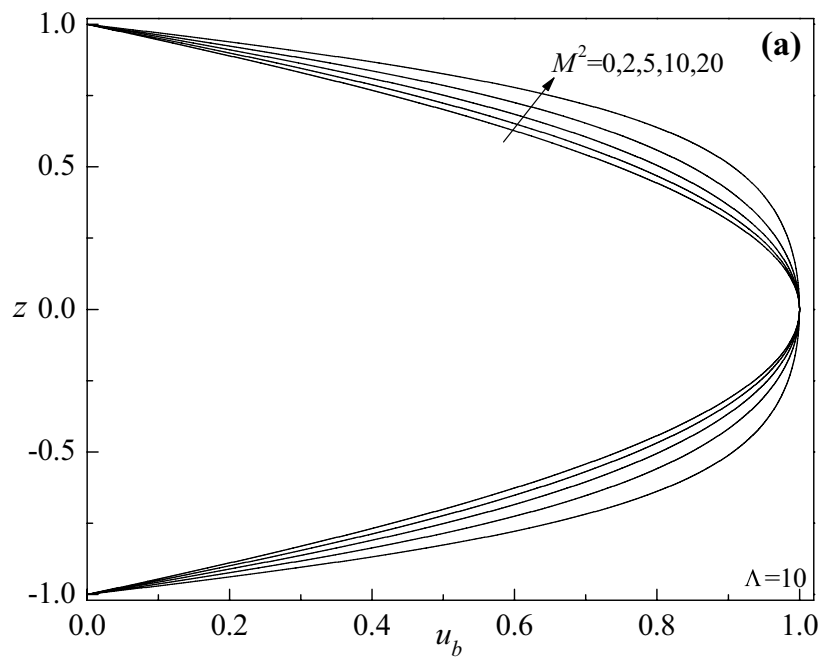

Figure 4 displays the neutral stability curves in the $(R, a)$ - plane showing the division of the plane into stable and unstable zones. The neutral stability curve is obtained when $M \rightarrow 0$ (hydrodynamic case) and $\Lambda \rightarrow \infty$ (absence of couple stresses) (Fig. 4a) from the present code and it can be seen that $R_{c}=5772.22$ and $a_{c}=1.020$, which are the known standard results (Orszag [42]). The computed values of $R_{c}$ for several values of $M$ as $\Lambda \rightarrow \infty$ are compared in Fig. $4 \mathrm{~b}$ with those of Takashima [4] and the outcomes complement with one another. These cross checking of results inspire further assurance in the prophecy of our numerical procedure.

The neutral stability curves for various values of Hartman number and couple stress parameter are exhibited,

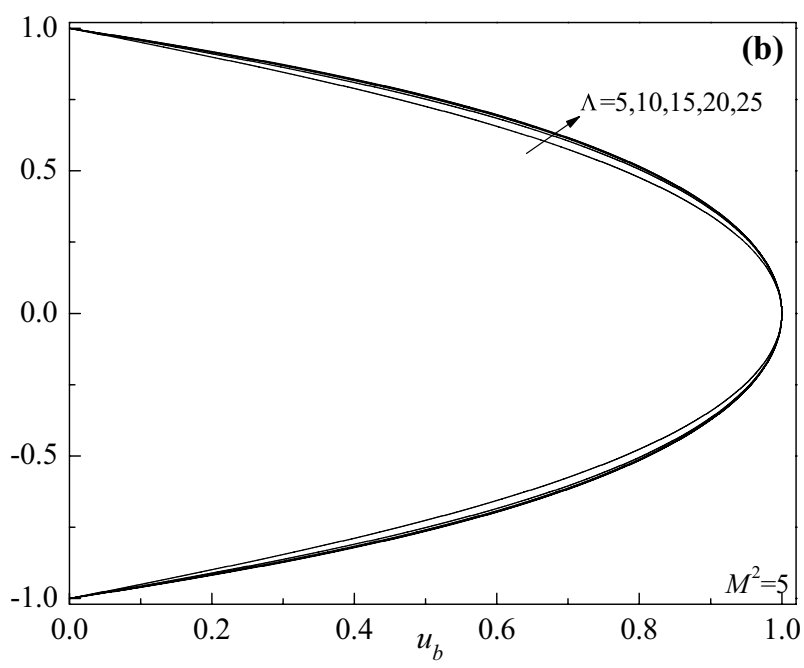

Fig. 2 Basic velocity profiles for different values of $\mathbf{a}$ Hartmann number and $\mathbf{b}$ couple stress parameter 

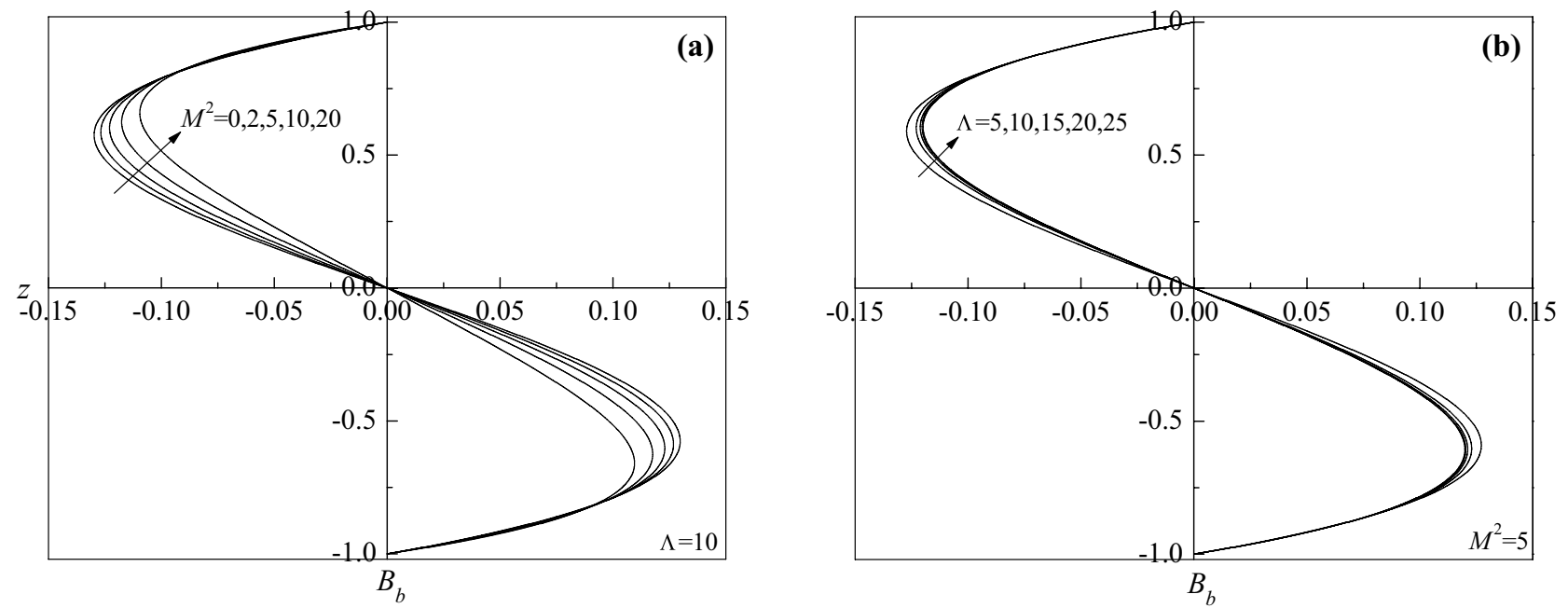

Fig. 3 Basic magnetic field profiles for different values of $\mathbf{a}$ Hartmann number and $\mathbf{b}$ couple stress parameter

respectively, in Fig. 4c, d. We observe that increasing $M$ is to increase $R_{c}$, indicating that the Hartman number enhances the stability of the system (Fig. 4c), whereas a dual behavior is observed with increasing $\Lambda$ (Fig. $4 d$ ). We locate that $R_{c}$ move towards higher values of $a$ with increasing $M$ representing that the cell width diminishes. However, the cell width enlarges with increasing $\Lambda$.

Figure 5 a illustrates the variation of $R_{c}$ as a function of $\Lambda$ for various values of $M$. When $M=0$, the curve emanates from $R_{c}=45695.77$ and experiences a steep fall as the value of $\Lambda$ increases to 6 . As the curve approaches to 20 , the gradient of the curve changes from negative to positive. The curve progresses with a gradually increasing positive gradient thereafter. The curve of $M=\sqrt{2}$ has a value of $R_{c}=11179.47$ at $\Lambda=2.9$. The curve faces a negative gradient initially and thereafter progresses with a gradually increasing positive gradient. The curves of $M^{2}=5,10,20$ and 25 follow a similar trend throughout the domain of $\Lambda$ considered. The curve of $M=0$ lies well below the curves of $M \neq 0$ indicating the magnetic field is more effective in stabilizing the system. This is due to the Lorentz force seizes backing the flow and therefore higher Reynolds number is necessary to coerce the flow. It is further seen that $R_{c}$ decrease with increasing value of $\Lambda$ initially for each $M$ due to decrease in the couple stress viscosity and thus it has a destabilizing effect on the flow. However, $R_{c}$ increases at higher values of $\Lambda$ as it turns to Newtonian case.

Figure $5 b$ establishes a relationship between $a_{c}$ and $\Lambda$ for different values of $M$. The curve of $M=0$ emanates from $a_{c}=1.604$ when $\Lambda=1$ and it progresses with a decreasing value of $a_{c}$ for increasing value of $\Lambda$. The curve of $M^{2}=2$ runs parallel to the curve of $M=0$ but originates from $(2.9,1.538)$. The curve of $M^{2}=5$ emanates from $(4.5,1.480)$ and has a constant negative gradient throughout where the value of $a_{c}$ decreases with increasing value of $\Lambda$. The curves of $M^{2}=10,20$ and 25 follow a similar trend with a positive gradient at the beginning followed by a gradual change to negative gradient, that is, the value of $a_{c}$ increases initially and then gradually decreases as the curve progresses. The corresponding critical wave speeds are illustrated in Fig. $5 \mathrm{c}$ as a function of $\Lambda$ for various values of $M$. The curves of $M^{2}=0,2,5,10,20$ and 25 follow a similar trend where they experience a gradual fall in their value as they progress. The curves for higher values of $M$ originate with a smaller value initially and the curves run almost parallel to each other. All the curves have their maximum value at their respective origin.

To obtain a clear understanding of the fluid flow stability characteristics for specific parameters, the secondary flow energy spectra are examined in detail. Theoretically at the critical points, $\partial E_{k i n} / \partial t=0$. Of all the numerical results produced here for the energy analysis, 
Fig. 4 Neutral stability curves
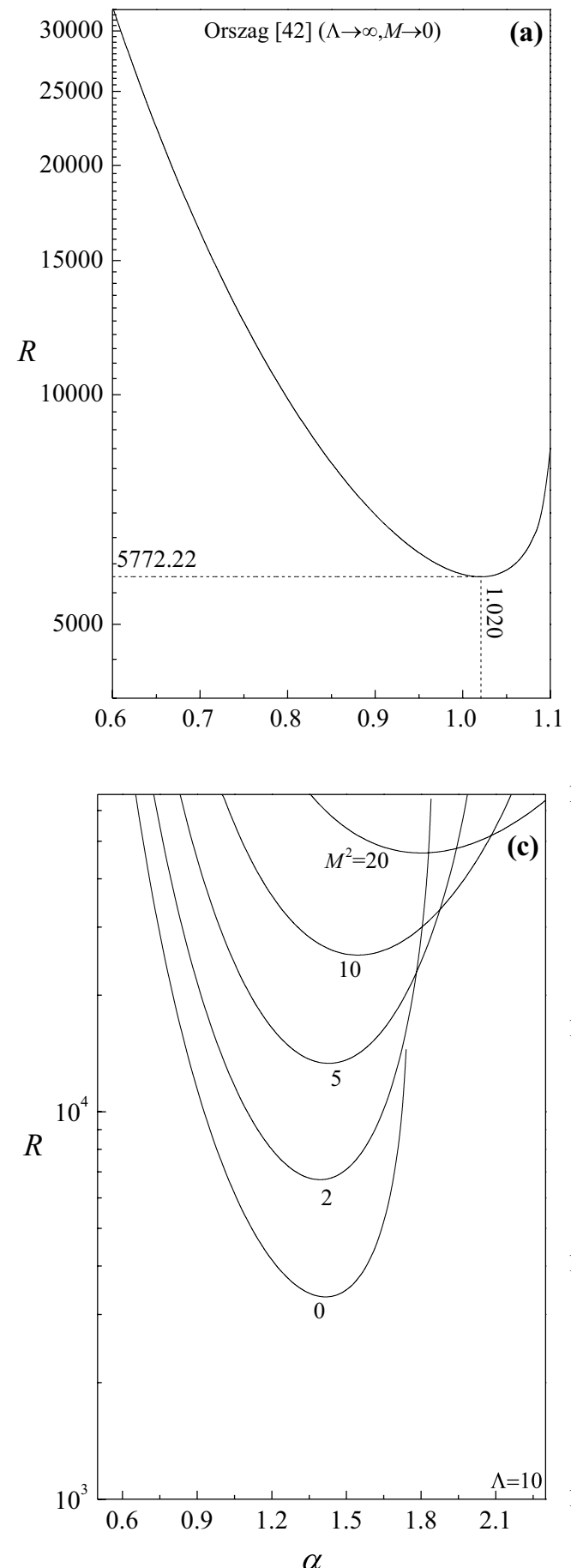
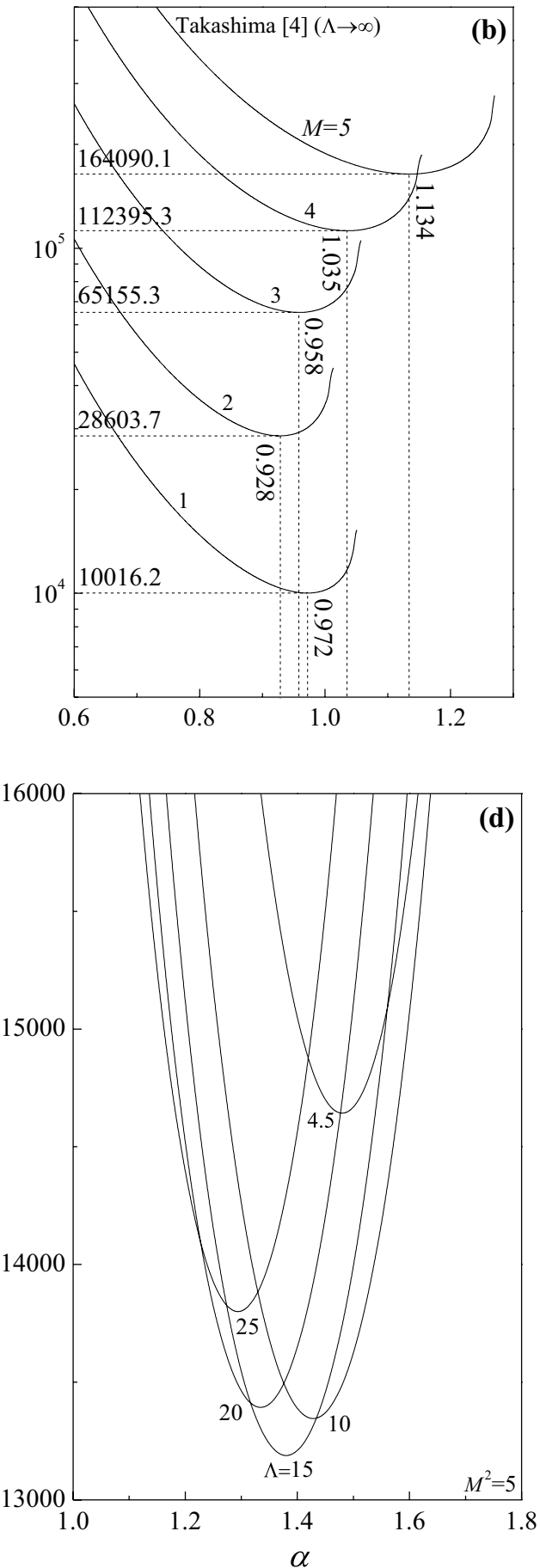

this phenomenon can be observed at the critical points. It can be seen that for all the values of $M$ considered here, $E_{s}$ has a propensity to destabilize the flow whereas $E_{d}, E_{c}$ and $E_{M}$ stabilize it, throughout the domain of couple stress parameter. However, the contribution of $E_{M}$ is negligible in the domain. One important finding of this study is that prior to the threshold value of couple stress parameter, the contribution of $E_{c}$ to stabilize the 

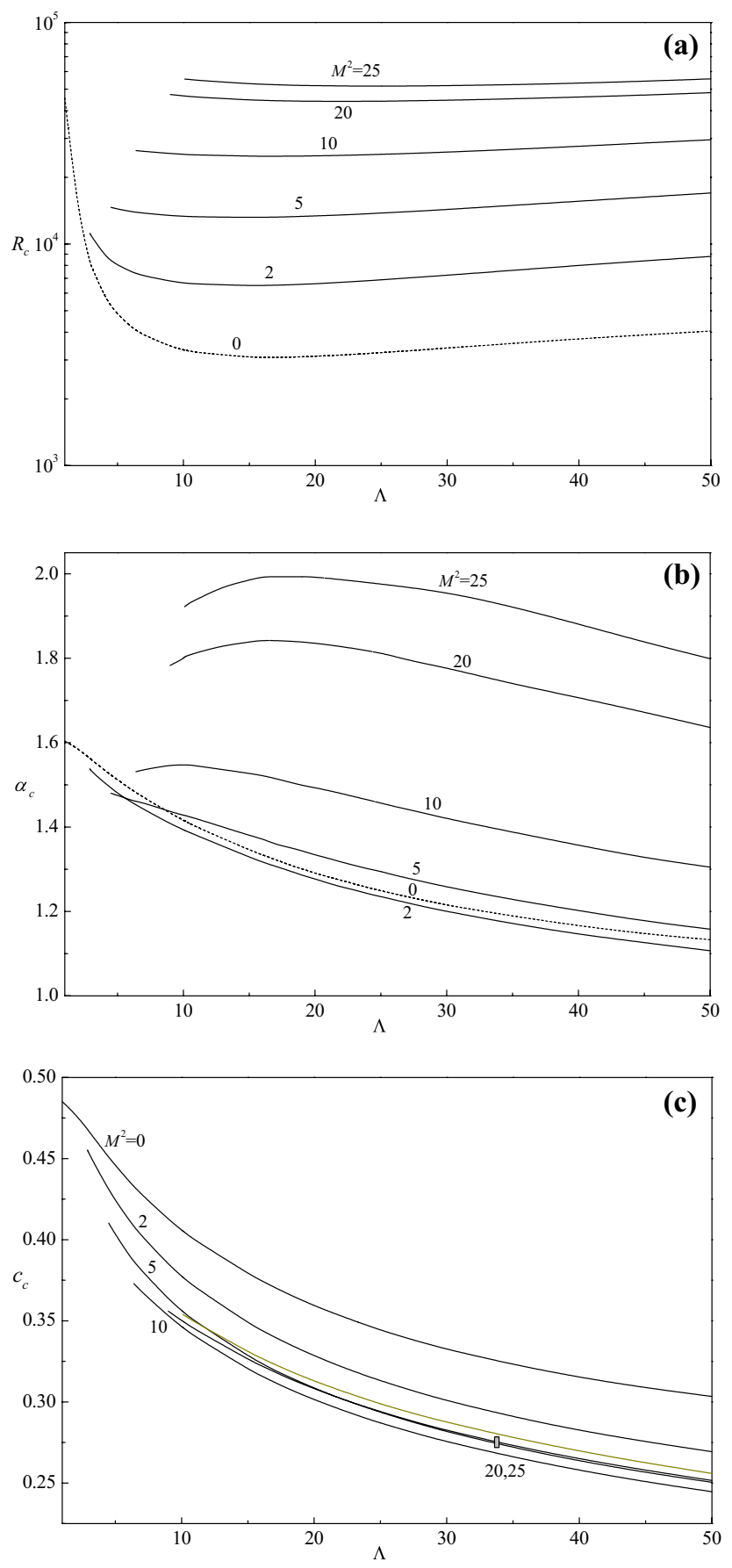

Fig. 5 Variation of a critical Reynolds number $R_{c}$ b critical wave number $a_{c}$ and $c$ critical wave speed $c_{c}$ with couple stress parameter $\Lambda$ for various values of Hartmann number $M$ flow is more than offered by $E_{d}$ but beyond the threshold value this characteristic reverses. Threshold values of couple stress parameter from where this reversal observed is shown in Fig. 6a-d. Quantitatively, prior to the threshold value of couple stress parameter, contribution of $E_{c}\left(E_{d}\right)$ in the stability of the fluid flow varies from 78 to $48 \%$ ( 19 to $48 \%$ ). It is also noted that the contribution of $E_{d}\left(E_{c}\right)$ is strictly increasing (decreasing) as a function of couple stress parameter.

\section{Conclusions}

The stability of forced convective flow is investigated in a horizontal channel of electrically conducting couple stress fluid under the influence of a uniform vertical magnetic field. The complicated complex stability equation is obtained and solved computationally by using the Chebyshev collocation method after determining that two-dimensional motions are more unstable than threedimensional motions via an analogue of Squire's transformation. The existence of a point in the basic flow field, for which the couple stress parameter must be greater than twice the Hartman number, is a necessary condition to be noted in order to exacerbate a disturbance. The Hartman number significantly affects the flow and increase in its value results in the stabilization of the fluid flow while increase in the couple stress parameter shows a twofold behavior. Energy spectrum indicates that kinetic energy due to the shear term $\left(E_{s}\right)$ has a propensity to destabilize the flow while kinetic energy due to surface drag $\left(E_{d}\right)$ and couple stress viscosity $\left(E_{c}\right)$ stabilizes it, throughout the domain of couple stress parameter. Nevertheless, the contribution of kinetic energy due to magnetic field $\left(E_{M}\right)$ is found to be negligible. Contribution of $E_{c}$ on the stability of flow is observed to be more (less) than $E_{d}$ prior (beyond) the threshold value of couple stress parameter. Also noted that the contribution of $E_{d}\left(E_{c}\right)$ is strictly increasing (decreasing) as a function of couple stress parameter. Finally, it is observed that the transverse magnetic field has a stabilizing effect on the Poiseuille flow even in the presence of couple stresses. 
Fig. 6 The rate of change of kinetic energy as a function of $\Lambda$ when $\mathbf{a} M^{2}=5 \mathbf{b} M^{2}=10 \mathbf{c}$ $M^{2}=20$ d $M^{2}=30$
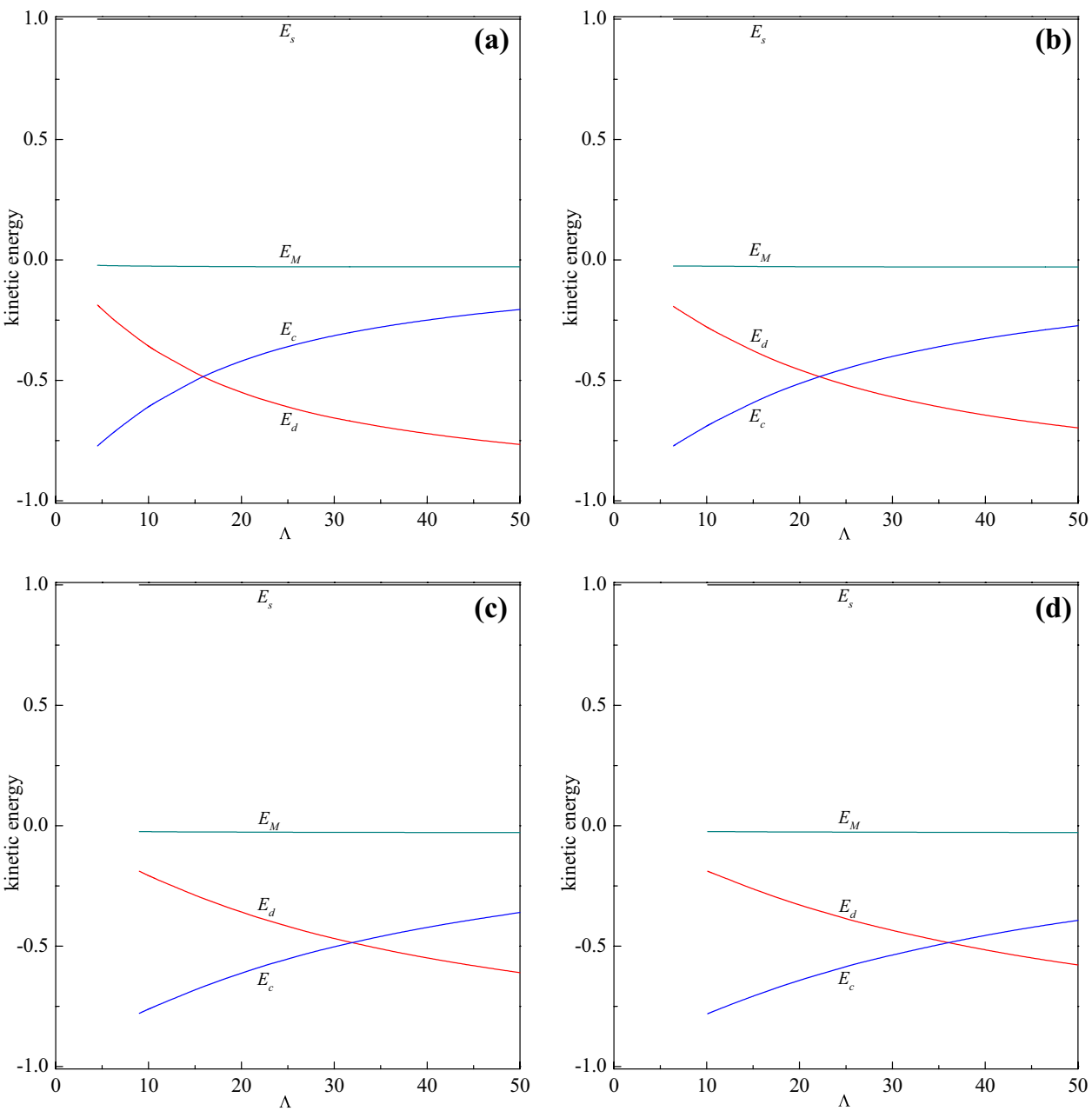

Acknowledgements The authors would like to acknowledge the institutions of their work for support. The authors are grateful to the referees for their most valuable comments that improved the paper considerably.

\section{Compliance with ethical standards}

Conflict of interest On behalf of all authors, the corresponding author states that there is no conflict of interest.

\section{References}

1. Drazin PG, Reid WH (2004) Hydrodynamic stability. Cambridge University Press, Cambridge

2. Lock RC (1955) The stability of the flow of an electrically conducting fluid between parallel planes under a transverse magnetic field. Proc R Soc Lond Ser A Math Phys Sci 233:105-125. https://doi.org/10.1098/rspa.1955.0249

3. Potter MC, Kutchey JA (1973) Stability of plane Hartmann flow subject to a transverse magnetic field. Phys Fluids 16:18481851. https://doi.org/10.1063/1.1694224

4. Takashima M (1996) The stability of the modified plane Poiseuille flow in the presence of a transverse magnetic field. Fluid Dyn Res 17:293-310. https://doi.org/10.1016/0169-5983(95)00038-0
5. Lingwood RJ, Alboussière $T$ (1999) On the stability of the Hartmann layer. Phys Fluids 11:2058-2068. https://doi. org/10.1063/1.870068

6. Makinde OD (2003) Magneto-hydrodynamic stability of planePoiseuille flow using multideck asymptotic technique. Math Comput Model 37:251-259. https://doi.org/10.1016/S0895 -7177(03)00004-9

7. Proskurin AV, Sagalakov AM (2008) Stability of Poiseuille flow in the presence of a longitudinal magnetic field. J Appl Mech Tech Phys 49:383-390. https://doi.org/10.1007/s10808-008-0053-z

8. Makinde OD, Mhone PY (2009) On temporal stability analysis for hydromagnetic flow in a channel filled with a saturated porous medium. Flow Turbul Combust 83:21-32. https://doi. org/10.1007/s10494-008-9187-6

9. Shankar BM, Shivakumara IS (2018) Magnetohydrodynamic stability of pressure-driven flow in an anisotropic porous channel: accurate solution. Appl Math Comput 321:752-767. https://doi. org/10.1016/j.amc.2017.11.006

10. Takashima M (1994) The stability of natural convection in a vertical layer of electrically conducting fluid in the presence of a transverse magnetic field. Fluid Dyn Res 14:121-134. https:// doi.org/10.1016/0169-5983(94)90056-6

11. Kaddeche S, Henry D, Benhadid H (2003) Magnetic stabilization of the buoyant convection between infinite horizontal walls with a horizontal temperature gradient. J Fluid Mech 480:185216. https://doi.org/10.1017/S0022112002003622 
12. Belyaev AV, Smorodin BL (2010) The stability of ferrofluid flow in a vertical layer subject to lateral heating and horizontal magnetic field. J Magn Magn Mater 322:2596-2606. https://doi. org/10.1016/j.jmmm.2010.03.028

13. Adesanya SO, Oluwadare EO, Falade JA, Makinde OD (2015) Hydromagnetic natural convection flow between vertical parallel plates with time-periodic boundary conditions. J Magn Magn Mater 396:295-303. https://doi.org/10.1016/j. jmmm.2015.07.096

14. Hayat T, Imtiaz M, Alsaedi A, Kutbi MA (2015) MHD threedimensional flow of nanofluid with velocity slip and nonlinear thermal radiation. J Magn Magn Mater 396:31-37. https://doi. org/10.1016/j.jmmm.2015.07.091

15. Hudoba A, Molokov S, Aleksandrova S, Pedcenko A (2016) Linear stability of buoyant convection in a horizontal layer of an electrically conducting fluid in moderate and high vertical magnetic field. Phys Fluids 28:094104. https://doi.org/10.1063/1.4962741

16. Hudoba A, Molokov S (2016) Linear stability of buoyant convective flow in a vertical channel with internal heat sources and a transverse magnetic field. Phys Fluids 28:114103. https://doi. org/10.1063/1.4965448

17. Bhatti MM, Abbas MA, Rashidi MM (2016) Combine effects of magnetohydrodynamics (MHD) and partial slip on peristaltic blood flow of Ree-Eyring fluid with wall properties. Eng Sci Technol Int J 19:1497-1502. https://doi.org/10.1016/j.jestc h.2016.05.004

18. Shankar BM, Kumar J, Shivakumara IS (2014) Stability of natural convection in a vertical couple stress fluid layer. Int J Heat Mass Transf 78:447-459. https://doi.org/10.1016/j.ijheatmasstrans fer.2014.06.087

19. Ijaz N, Bhatti M, Zeeshan A (2019) Heat transfer analysis in magnetohydrodynamic flow of solid particles in non-Newtonian Ree-Eyring fluid due to peristaltic wave in a channel. Therm Sci 23:1017-1026. https://doi.org/10.2298/tsci170220155i

20. Hassan M, Ellahi R, Bhatti MM, Zeeshan A (2019) A comparative study on magnetic and non-magnetic particles in nanofluid propagating over a wedge. Can J Phys 97:277-285. https://doi. org/10.1139/cjp-2018-0159

21. Shankar BM, Kumar J, Shivakumara IS (2019) Magnetohydrodynamic instability of mixed convection in a differentially heated vertical channel. Eur Phys J Plus 134:53. https://doi.org/10.1140/ epjp/i2019-12402-0

22. Stokes VK (1966) Couple stresses in fluids. Phys Fluids 9:17091715. https://doi.org/10.1063/1.1761925

23. Jain JK, Stokes VK (1972) Effects of couple stresses on the stability of plane poiseuille flow. Phys Fluids 15:977-980. https://doi. org/10.1063/1.1694059

24. Shankar BM, Shivakumara IS, Ng CO (2016) Stability of couple stress fluid flow through a horizontal porous layer. J Porous Media 19:391-404. https://doi.org/10.1615/JPorMedia.v19.i5.20

25. Mekheimer KS (2008) Effect of the induced magnetic field on peristaltic flow of a couple stress fluid. Phys Lett A 372:42714278. https://doi.org/10.1016/j.physleta.2008.03.059

26. Shankar BM, Kumar J, Shivakumara IS (2016) Stability of natural convection in a vertical dielectric couple stress fluid layer in the presence of a horizontal AC electric field. Appl Math Model 40:5462-5481. https://doi.org/10.1016/j.apm.2016.01.005

27. Shankar BM, Kumar J, Shivakumara IS, Raghunatha KR (2018) Stability of natural convection in a vertical non-Newtonian fluid layer with an imposed magnetic field. Meccanica 53:773-786. https://doi.org/10.1007/s11012-017-0770-6

28. Rudraiah N, Shankar BM, Ng CO (2011) Electrohydrodynamic stability of couple stress fluid flow in a channel occupied by a porous medium. Spec Top Rev Porous Media 2:11-22. https:// doi.org/10.1615/SpecialTopicsRevPorousMedia.v2.i1.20

29. Shivakumara IS, Akkanagamma M, Ng CO (2013) Electrohydrodynamic instability of a rotating couple stress dielectric fluid layer. Int J Heat Mass Transf 62:761-771. https://doi. org/10.1016/j.ijheatmasstransfer.2013.03.050

30. Turkyilmazoglu M (2014) Exact solutions for two-dimensional laminar flow over a continuously stretching or shrinking sheet in an electrically conducting quiescent couple stress fluid. Int J Heat Mass Transf 72:1-8. https://doi.org/10.1016/j.ijheatmass transfer.2014.01.009

31. Shivakumara IS, Naveen Kumar SB (2014) Linear and weakly nonlinear triple diffusive convection in a couple stress fluid layer. Int J Heat Mass Transf 68:542-553. https://doi.org/10.1016/j.ijhea tmasstransfer.2013.09.051

32. Srinivasacharya D, Rao GM (2016) Computational analysis of magnetic effects on pulsatile flow of couple stress fluid through a bifurcated artery. Comput Methods Progr Biomed 137:269279. https://doi.org/10.1016/j.cmpb.2016.09.015

33. Ramesh K (2016) Influence of heat and mass transfer on peristaltic flow of a couple stress fluid through porous medium in the presence of inclined magnetic field in an inclined asymmetric channel. J Mol Liq 219:256-271. https://doi.org/10.1016/j.molli q.2016.03.010

34. Tripathi D, Yadav A, Anwar Bég O (2017) Electro-osmotic flow of couple stress fluids in a micro-channel propagated by peristalsis. Eur Phys J Plus. https://doi.org/10.1140/epjp/i2017-11416-x

35. Mahabaleshwar US, Sarris IE, Hill AA et al (2017) An MHD couple stress fluid due to a perforated sheet undergoing linear stretching with heat transfer. Int J Heat Mass Transf 105:157-167. https ://doi.org/10.1016/j.ijheatmasstransfer.2016.09.040

36. Layek GC, Pati NC (2018) Chaotic thermal convection of couple-stress fluid layer. Nonlinear Dyn 91:837-852. https://doi. org/10.1007/s11071-017-3913-3

37. Nandal R, Mahajan A (2018) Penetrative convection in couplestress fluid via internal heat source/sink with the boundary effects. J Nonnewton Fluid Mech 260:133-141. https://doi. org/10.1016/j.jnnfm.2018.07.004

38. Janardhana Reddy G, Kumar M, Kethireddy B, Chamkha AJ (2018) Colloidal study of unsteady magnetohydrodynamic couple stress fluid flow over an isothermal vertical flat plate with entropy heat generation. J Mol Liq 252:169-179. https://doi. org/10.1016/j.molliq.2017.12.106

39. Naveen Kumar SB, Shivakumara IS, Shankar BM (2019) Exploration of coriolis force on the linear stability of couple stress fluid flow induced by double diffusive convection. J Heat Transf. https ://doi.org/10.1115/1.4044699

40. Canuto C, Hussaini MY, Quarteroni A, Zang TA (1988) Spectral methods in fluid dynamics. Springer, Berlin

41. Shankar BM, Kumar J, Shivakumara IS (2019) Stability of mixed convection in a differentially heated vertical fluid layer with internal heat sources. Fluid Dyn Res 51:055501. https://doi. org/10.1088/1873-7005/ab2d50

42. Orszag SA (1971) Accurate solution of the Orr-Sommerfeld stability equation. J Fluid Mech 50:689-703. https://doi. org/10.1017/S0022112071002842

Publisher's Note Springer Nature remains neutral with regard to jurisdictional claims in published maps and institutional affiliations. 